\title{
On strengthened form of Copson's inequality
}

Qian Xu

"Correspondence:

xq32153215@yahoo.com.cn

Jiaxing Radio \& TV University,

Jiaxing, Zhejiang 314000, P.R. China

\section{Abstract}

In this paper, the famous Copson inequality has been improved. We obtain some new results by a different method.

MSC: 26D15; 25D05

Keywords: inequality; Copson's inequality; analytic inequalities

\section{Introduction}

Suppose that $a_{k}>0, p>1$, then we obtain the following Hardy inequality:

$$
\left(\frac{p}{p-1}\right)^{p} \sum_{n=1}^{\infty} a_{n}^{p}>\sum_{n=1}^{\infty}\left(\frac{1}{n} \sum_{k=1}^{n} a_{k}\right)^{p} .
$$

Hardy's inequality plays an important role in the field of analysis; see [1-4]. In recent decades, some generalizations and strengthening of Hardy's inequality have been obtained in $[1-5]$. We list some previous results as follows.

Copson's inequality $[4,5]$ Suppose that $a_{n}>0(n=1,2,3 \ldots)$. If $p>1$, then

$$
\sum_{n=1}^{\infty}\left(\sum_{k=n}^{\infty} \frac{a_{k}}{k}\right)^{p}<p^{p} \sum_{n=1}^{\infty} a_{n}^{p} .
$$

If $0<p<1$, then

$$
\sum_{n=1}^{\infty}\left(\sum_{k=n}^{\infty} \frac{a_{k}}{k}\right)^{p}>p^{p} \sum_{n=1}^{\infty} a_{n}^{p} .
$$

And in [4] and [6], the authors pay much attention to the generalization of Copson's inequality.

In this paper, inequalities (1.2) and (1.3) were strengthened by using a new method.

\section{Relational lemmas and definitions}

In this section, some relational lemmas and definitions will be introduced.

Theorem A [7, Th. 1.1] Suppose that $a, b \in \mathbb{R}, a<b, c \in[a, b]$, and $f:[a, b]^{n} \rightarrow \mathbb{R}$ has continuous partial derivatives, and

$$
D_{i}=\left\{\left(x_{1}, x_{2}, \ldots, x_{n-1}, c\right) \mid \min _{1 \leq k \leq n-1}\left\{x_{k}\right\} \geq c, x_{i}=\min _{1 \leq k \leq n-1}\left\{x_{k}\right\} \neq c\right\}, \quad i=1,2, \ldots, n-1 .
$$

(0) 2012 Xu; licensee Springer. This is an Open Access article distributed under the terms of the Creative Commons Attribution License (http://creativecommons.org/licenses/by/2.0), which permits unrestricted use, distribution, and reproduction in any medium, provided the original work is properly cited. 
If $\frac{\partial f(x)}{\partial x_{i}}>0$ holds for all $x \in D_{i}(i=1,2, \ldots, n-1)$, then

$$
f\left(y_{1}, y_{2}, \ldots, y_{n-1}, c\right) \geq f(c, c, \ldots, c, c)
$$

where $y_{i} \in[c, b](i=1,2, \ldots, n-1)$.

Theorem B [7, Cor. 1.3] Suppose that $a, b \in \mathbb{R}, a<b$, and $f:[a, b]^{n} \rightarrow \mathbb{R}$ has continuous partial derivatives and

$$
D_{i}=\left\{\left(x_{1}, x_{2}, \ldots, x_{n}\right) \mid a \leq \min _{1 \leq k \leq n}\left\{x_{k}\right\}<x_{i}=\max _{1 \leq k \leq n}\left\{x_{k}\right\} \leq b\right\}, \quad i=1,2, \ldots, n .
$$

If $\frac{\partial f(x)}{\partial x_{i}}>0$ holds for all $x \in D_{i}$, where $i=1,2, \ldots, n$, then

$$
f\left(x_{1}, x_{2}, \ldots, x_{n}\right) \geq f\left(x_{\min }, x_{\min }, \ldots, x_{\min }\right),
$$

where $x_{i} \in[a, b](i=1,2, \ldots, n), x_{\min }=\min _{1 \leq k \leq n}\left\{x_{k}\right\}$.

Definition 1 [1] Let $G \subseteq \mathbb{R}^{n}$ be a convex set, $\phi: H \rightarrow \mathbb{R}$ be a continuous function. If

$$
\phi(\alpha x+(1-\alpha) y) \leq(\geq) \alpha \phi(x)+(1-\alpha) \phi(y)
$$

holds for all $x, y \in G, \alpha \in[0,1]$, then the function $\varphi$ is convex (concave).

Lemma 1 (Hermite-Hadamard's inequality) Let $\phi:[a, b] \rightarrow \mathbb{R}$ be a convex (concave) function. Then

$$
\phi\left(\frac{a+b}{2}\right) \leq(\geq) \frac{1}{b-a} \int_{a}^{b} \phi(x) d x \leq(\geq) \frac{\phi(a)+\phi(b)}{2}
$$

and the equality holds if and only if $\phi$ is linear.

Lemma 2 Suppose that $p>0$.

(1) If $p \geq 1$, or $0<p \leq \frac{1}{2}$, then

$$
2 p^{p} \geq 2^{p}
$$

(2) If $\frac{1}{2}<p<1$, then

$$
2^{p}>2 p^{p}
$$

(3) If $\frac{1}{2}<p<1$, then

$$
p^{p}+(p-1) 2^{p}>0
$$

Proof Set $f_{1}: p \in(0,+\infty) \rightarrow p \ln p+\ln 2-p \ln 2$. Then we have

$$
f_{1}^{\prime}(p)=\ln p+1-\ln 2=\ln \left(\frac{e p}{2}\right)
$$


Obviously, $f_{1}$ is monotone decreasing for $p \in\left(0, \frac{2}{e}\right), f_{1}$ is monotone increasing for $p \in$ $\left(\frac{2}{e},+\infty\right)$, and $f_{1}(1)=0, f_{1}\left(\frac{1}{2}\right)=-\frac{1}{2} \ln 2+\ln 2-\frac{1}{2} \ln 2=0$, then (2.2) and (2.3) hold. Let

$$
f_{2}: p \in(0,1) \rightarrow p \ln p-p \ln 2-\ln (1-p)
$$

We get

$$
\begin{aligned}
& f_{2}^{\prime}(p)=\frac{1}{1-p}[(1-p) \ln p+(1-p)(1-\ln 2)+1] \stackrel{\text { Def. }}{=} \frac{1}{1-p} h(p) \\
& h^{\prime}(p)=-\ln p+\frac{1}{p}-2+\ln 2
\end{aligned}
$$

and

$$
h^{\prime \prime}(p)=-\frac{1}{p}-\frac{1}{p^{2}}<0
$$

Then $h$ is concave for $p \in(0,1)$. Because $h\left(\frac{1}{2}\right)>0$ and $\lim _{p \rightarrow 1^{-}} h(p)>0$, then $h(p)>0$ and $f_{2}^{\prime}(p)>0$ hold for $p \in\left[\frac{1}{2}, 1\right)$. From $f_{2}\left(\frac{1}{2}\right)=0$, we have $f_{2}(p)>0$ for $p \in\left(\frac{1}{2}, 1\right)$. Inequality (2.4) is proved.

\section{Lemma 3}

(1) If $p>1$, then the equation

$$
p^{p}(1-x)\left(\frac{1}{2}-x\right)^{p-1}=1
$$

has only a positive root for $x \in\left(0, \frac{1}{2}\right)$.

(2) If $\frac{1}{2}<p<1$, then the equation

$$
p^{p}(1+x)=\left(\frac{1}{2}+x\right)^{1-p}
$$

has only a positive root for $x \in\left(0, \frac{1}{2}\right)$.

Proof

(1) Let $g_{1}: x \in\left[0, \frac{1}{2}\right] \rightarrow p^{p}(1-x)\left(\frac{1}{2}-x\right)^{p-1}-1$. Then $g_{1}$ is monotone decreasing. According to inequality (2.2), we have

$$
g_{1}(0)=p^{p}\left(\frac{1}{2}\right)^{p-1}-1=\left(\frac{1}{2}\right)^{p-1}\left[p^{p}-2^{p-1}\right]>0
$$

and $g_{1}\left(\frac{1}{2}\right)=-1$. So, equation (2.5) has only a positive root for $x \in\left(0, \frac{1}{2}\right)$.

(2) Let $g_{2}: x \in\left[0, \frac{1}{2}\right] \rightarrow p^{p}(1+x)-\left(\frac{1}{2}+x\right)^{1-p}$. Thus,

$$
g_{2}^{\prime}(x)=p^{p}-(1-p)\left(\frac{1}{2}+x\right)^{-p}>\left(\frac{1}{2}+x\right)^{-p}\left[\frac{1}{2^{p}} \cdot p^{p}-(1-p)\right]
$$


By inequality (2.4), $g_{2}$ is monotone increasing. According to inequality (2.3), we get

$$
\lim _{x \rightarrow 0^{+}} g_{2}(x)=p^{p}-\frac{1}{2^{1-p}}<0
$$

and

$$
\lim _{x \rightarrow(1 / 2)^{+}} g_{2}(x)=\frac{3}{2} p^{p}-1 \geq \frac{3}{2} \cdot\left(\frac{1}{2}\right)^{\frac{1}{2}}-1>0 .
$$

Therefore, equation (2.6) has only a positive root for $x \in\left(0, \frac{1}{2}\right)$.

Lemma 4 If $p>1, m>0, m \in \mathrm{N}$ and $c \in\left(0, \frac{1}{2}\right)$ is the only one positive root of equation (2.5), then

$$
\sum_{n=1}^{m}\left[\sum_{k=n}^{\infty} \frac{1}{(k-c)^{1+\frac{1}{p}}}\right]^{p-1}<p^{p}(m-c)^{\frac{1}{p}}
$$

and

$$
\sum_{n=1}^{m}\left[\sum_{k=n}^{m} \frac{1}{(k-c)^{1+1 / p}}\right]^{p}<p^{p} \sum_{n=1}^{m} \frac{n^{p}}{(n-c)^{p+1}} .
$$

Proof (1) If $m=1$, by Lemma 1 , we get

$$
\begin{aligned}
\sum_{n=1}^{m}\left[\sum_{k=n}^{\infty} \frac{1}{(k-c)^{1+1 / p}}\right]^{p-1} & =\left[\sum_{k=1}^{\infty} \frac{1}{(k-c)^{1+1 / p}}\right]^{p-1} \\
& <\left[\int_{\frac{1}{2}}^{\infty} \frac{1}{(x-c)^{1+1 / p}} d x\right]^{p-1}=p^{p-1}\left(\frac{1}{2}-c\right)^{-(p-1) / p} .
\end{aligned}
$$

If $m \geq 2$, by Lemma 1 , we get

$$
\begin{aligned}
\sum_{n=1}^{m}\left[\sum_{k=n}^{\infty} \frac{1}{(k-c)^{1+1 / p}}\right]^{p-1} & <\sum_{n=1}^{m}\left[\int_{n-\frac{1}{2}}^{\infty} \frac{1}{(x-c)^{1+1 / p}}\right]^{p-1} \\
& =p^{p-1} \sum_{n=1}^{m}\left(n-\frac{1}{2}-c\right)^{-(p-1) / p} \\
& =p^{p-1}\left[\left(\frac{1}{2}-c\right)^{-(p-1) / p}+\sum_{n=2}^{m}\left(n-\frac{1}{2}-c\right)^{-(p-1) / p}\right] \\
& <p^{p-1}\left[\left(\frac{1}{2}-c\right)^{-(p-1) / p}+\int_{\frac{3}{2}}^{m+\frac{1}{2}}\left(x-\frac{1}{2}-c\right)^{-(p-1) / p} d x\right] \\
& =p^{p-1}\left[\left(\frac{1}{2}-c\right)^{-(p-1) / p}+p(m-c)^{1 / p}-p(1-c)^{1 / p}\right] .
\end{aligned}
$$


So,

$$
\sum_{n=1}^{m}\left[\sum_{k=n}^{\infty} \frac{1}{(k-c)^{1+1 / p}}\right]^{p-1}<p^{p-1}\left[\left(\frac{1}{2}-c\right)^{-(p-1) / p}+p(m-c)^{1 / p}-p(1-c)^{1 / p}\right]
$$

holds for every $m>0$ and $m \in$ N. Since inequalities (2.9), (2.10) and

$$
\left(\frac{1}{2}-c\right)^{-(p-1) / p}=p(1-c)^{1 / p}
$$

inequality (2.7) holds.

(2)

$$
\begin{aligned}
& \sum_{n=1}^{m}\left[\sum_{k=n}^{m} \frac{1}{(k-c)^{1+1 / p}}\right]^{p} \\
& =p \sum_{n=1}^{m} \int_{0}^{\sum_{k=n}^{m} \frac{1}{(k-c)^{1+1 / p}}} x^{p-1} d x \\
& =p \sum_{n=1}^{m}\left[\int_{0}^{\frac{1}{(m-c)^{1+1 / p}}} x^{p-1} d x+\int_{\frac{1}{(m-c)^{1+1 / p}}}^{\frac{1}{(m-c)^{1+1 / p}}+\frac{1}{(m-c-1)^{1+1 / p}}} x^{p-1} d x+\cdots\right. \\
& \left.+\int_{\sum_{k=n+1}^{m} \frac{1}{(k-c)^{1+1 / p}}}^{\sum_{k=n}^{m} \frac{1}{(k-c)^{1+1 / p}}} x^{p-1} d x\right] \\
& <p \sum_{n=1}^{m}\left[\frac{1}{(m-c)^{1+1 / p}} \cdot\left(\frac{1}{(m-c)^{1+1 / p}}\right)^{p-1}+\frac{1}{(m-c-1)^{1+1 / p}}\right. \\
& \left.\cdot\left(\frac{1}{(m-c)^{1+1 / p}}+\frac{1}{(m-c-1)^{1+1 / p}}\right)^{p-1}+\cdots+\frac{1}{(n-c)^{1+1 / p}}\left(\sum_{k=n}^{m} \frac{1}{(k-c)^{1+1 / p}}\right)^{p-1}\right] \\
& =p\left[\frac{m}{(m-c)^{1+1 / p}} \cdot\left(\frac{1}{(m-c)^{1+1 / p}}\right)^{p-1}+\frac{m-1}{(m-c-1)^{1+1 / p}}\left(\frac{1}{(m-c)^{1+1 / p}}\right.\right. \\
& \left.\left.+\frac{1}{(m-c-1)^{1+1 / p}}\right)^{p-1}+\cdots+\frac{1}{(1-c)^{1+1 / p}}\left(\sum_{k=1}^{m} \frac{1}{k^{1+1 / p}}\right)^{p-1}\right] \\
& =p \sum_{n=1}^{m} \sum_{k=n}^{m} \frac{1}{(k-c)^{1+1 / p}}\left(\sum_{i=k}^{m} \frac{1}{(i-c)^{1+1 / p}}\right)^{p-1}=p \sum_{n=1}^{m} \frac{n}{(n-c)^{1+1 / p}}\left(\sum_{k=n}^{m} \frac{1}{(k-c)^{1+1 / p}}\right)^{p-1} \text {. }
\end{aligned}
$$

Let $q>1$ and $1 / p+1 / q=1$. Using Hölder's inequality, we have

$$
\begin{aligned}
& \sum_{n=1}^{m}\left[\sum_{k=n}^{m} \frac{1}{(k-c)^{1+1 / p}}\right]^{p} \\
& \quad<p\left[\sum_{n=1}^{m}\left(\frac{n}{(n-c)^{1+1 / p}}\right)^{p}\right]^{1 / p} \cdot\left[\sum_{n=1}^{m}\left(\sum_{k=n}^{m} \frac{1}{(k-c)^{1+1 / p}}\right)^{(p-1) q}\right]^{1 / q} \\
& \quad=p\left[\sum_{n=1}^{m} \frac{n^{p}}{(n-c)^{p+1}}\right]^{1 / p} \cdot\left[\sum_{n=1}^{m}\left(\sum_{k=n}^{m} \frac{1}{(k-c)^{1+1 / p}}\right)^{1 / q} \cdot\right.
\end{aligned}
$$


Xu Journal of Inequalities and Applications 2012, 2012:305

Page 6 of 11

Since

$$
\left[\sum_{n=1}^{m}\left(\sum_{k=n}^{m} \frac{1}{(k-c)^{1+1 / p}}\right)^{p}\right]^{1 / p}<p\left[\sum_{n=1}^{m} \frac{n^{p}}{(n-c)^{p+1}}\right]^{1 / p},
$$

inequality (2.8) holds.

Lemma 5 If $\frac{1}{2}<p<1, m>0, m \in \mathrm{N}$ and $d \in\left(0, \frac{1}{2}\right)$ is the only one positive root of equation (2.6), then

$$
\sum_{n=1}^{m}\left[\sum_{k=n}^{m} \frac{1}{(k+d)^{1+1 / p}}\right]^{p}>p^{p} \sum_{n=1}^{m} \frac{n^{p}}{(n+d)^{p+1}}
$$

Proof

$$
\begin{aligned}
& \sum_{n=1}^{m}\left[\sum_{k=n}^{m} \frac{1}{(k+d)^{1+1 / p}}\right]^{p} \\
& =p \sum_{n=1}^{m} \int_{0}^{\sum_{k=n}^{m} \frac{1}{(k+d)^{1+1 / p}}} x^{p-1} d x \\
& =p \sum_{n=1}^{m}\left[\int_{0}^{\frac{1}{(m+d)^{1+1 / p}}} x^{p-1} d x+\int_{\frac{1}{(m+d)^{1+1 / p}}}^{\sum_{k=m-1}^{m} \frac{1}{(k+d)^{1+1 / p}}} x^{p-1} d x+\int_{\sum_{k=m-1}^{m} \frac{1}{(k+d)^{1+1 / p}}}^{\sum_{k=m-2}^{m} \frac{1}{(k+d)^{1+1 / p}}} x^{p-1} d x+\cdots\right.
\end{aligned}
$$

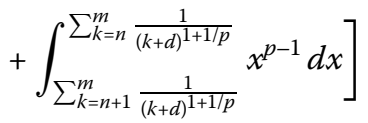

$$
\begin{aligned}
& >p \sum_{n=1}^{m}\left[\frac{1}{(m+d)^{1+1 / p}}\left(\frac{1}{(m+d)^{1+1 / p}}\right)^{-(1-p)}\right. \\
& +\frac{1}{(m-1+d)^{1+1 / p}}\left(\sum_{k=m-1}^{m} \frac{1}{(k+d)^{1+1 / p}}\right)^{-(1-p)}+\cdots \\
& \left.+\frac{1}{(n+d)^{1+1 / p}}\left(\sum_{k=n}^{m} \frac{1}{(k+d)^{1+1 / p}}\right)^{-(1-p)}\right] \\
& =p \sum_{n=1}^{m} \frac{n}{(n+d)^{1+1 / p}}\left(\sum_{k=n}^{m} \frac{1}{(k+d)^{1+1 / p}}\right)^{-(1-p)} \text {. }
\end{aligned}
$$

By Hölder's inequality, we have

$$
\begin{aligned}
& \sum_{n=1}^{m}\left[\left(\frac{n}{(n+d)^{1+1 / p}}\left(\sum_{k=n}^{m} \frac{1}{(k+d)^{1+1 / p}}\right)^{-(1-p)}\right)^{p} \cdot\left(\sum_{k=n}^{m} \frac{1}{(k+d)^{1+1 / p}}\right)^{p(1-p)}\right] \\
& <\left[\sum_{n=1}^{m}\left(\frac{n}{(n+d)^{1+1 / p}}\left(\sum_{k=n}^{m} \frac{1}{(k+d)^{1+1 / p}}\right)^{-(1-p)}\right)\right]^{p} \cdot\left[\sum_{n=1}^{m}\left(\sum_{k=n}^{m} \frac{1}{(k+d)^{1+1 / p}}\right)^{p}\right]^{1-p} .
\end{aligned}
$$


And by using inequality (2.12), we obtain

$$
\begin{aligned}
& \sum_{n=1}^{m} \frac{n}{(n+d)^{1+1 / p}}\left(\sum_{k=n}^{m} \frac{1}{(k+d)^{1+1 / p}}\right)^{-(1-p)} \cdot\left[\sum_{n=1}^{m}\left(\sum_{k=n}^{m} \frac{1}{(k+d)^{1+1 / p}}\right)^{p}\right]^{\frac{1-p}{p}} \\
& \quad>\left\{\sum_{n=1}^{m}\left[\left(\frac{n}{(n+d)^{1+1 / p}}\left(\sum_{k=n}^{m} \frac{1}{(k+d)^{1+1 / p}}\right)^{-(1-p)} \cdot\left(\sum_{k=n}^{m} \frac{1}{(k+d)^{1+1 / p}}\right)^{p(1-p)}\right]\right\}^{1 / p}\right. \\
& \quad=\left\{\sum_{n=1}^{m}\left[\frac{n^{p}}{(n+d)^{p+1}}\left(\sum_{k=n}^{m} \frac{1}{(k+d)^{1+1 / p}}\right)^{-(1-p) p} \cdot\left(\sum_{k=n}^{m} \frac{1}{(k+d)^{1+1 / p}}\right)^{1-p)}\right]\right\}^{1 / p} \\
& \quad=\left(\sum_{n=1}^{m} \frac{n^{p}}{(n+d)^{p+1}}\right)^{1 / p} .
\end{aligned}
$$

From inequality (2.12) and inequality (2.13), we get

$$
\sum_{n=1}^{m}\left(\sum_{k=n}^{m} \frac{1}{(k+d)^{1+1 / p}}\right)^{p}>p \frac{\left[\sum_{n=1}^{m} \frac{n^{p}}{(n+d)^{p+1}}\right]^{1 / p}}{\left\{\sum_{n=1}^{m}\left[\sum_{k=n}^{m} \frac{1}{(k+d)^{1+1 / p}}\right]^{p}\right\}^{\frac{1-p}{p}}}
$$

and

$$
\left\{\sum_{n=1}^{m}\left[\sum_{k=n}^{m} \frac{1}{(k+d)^{1+1 / p}}\right]^{p}\right\}^{1 / p}>p\left[\sum_{n=1}^{m} \frac{n^{p}}{(n+d)^{p+1}}\right]^{1 / p} .
$$

Then inequality (2.11) holds.

\section{Strengthened Copson's inequality $(p>1)$}

Theorem 1 Assume that $p>1, m>0, m \in \mathrm{N}, a_{n}>0(n=1,2, \ldots, m), c \in\left(0, \frac{1}{2}\right)$ is the only one positive root of equation (2.5) and $B_{m}=\min _{1 \leq n \leq m}\left\{(n-c)^{1 / p} a_{n}\right\}$. Then

$$
\begin{aligned}
& p^{p} \sum_{n=1}^{m} a_{n}^{p}-\sum_{n=1}^{m}\left(\sum_{k=n}^{m} \frac{a_{k}}{k-c}\right)^{p} \\
& \geq B_{m}^{p}\left[p^{p} \sum_{n=1}^{m} \frac{1}{n-c}-\sum_{n=1}^{m}\left(\sum_{k=n}^{m} \frac{1}{(k-c)^{1+1 / p}}\right)^{p}\right] .
\end{aligned}
$$

Proof Set $b_{n}=(n-c)^{1 / p} a_{n}(n=1,2, \ldots, m)$. Then inequality (3.1) is equivalent to

$$
\begin{aligned}
& p^{p} \sum_{n=1}^{m} \frac{b_{n}^{p}}{n-c}-\sum_{n=1}^{m}\left[\sum_{k=n}^{m} \frac{b_{k}}{(k-c)^{1+1 / p}}\right]^{p} \\
& \geq B_{m}^{p}\left[p^{p} \sum_{n=1}^{m} \frac{1}{n-c}-\sum_{n=1}^{m}\left(\sum_{k=n}^{m} \frac{1}{(k-c)^{1+1 / p}}\right)^{p}\right],
\end{aligned}
$$

where $B_{m}=\min _{1 \leq n \leq N}\left\{b_{n}\right\}$. Let

$$
f: b=\left(b_{1}, b_{2}, \ldots, b_{m}\right) \in[0,+\infty)^{m} \rightarrow p^{p} \sum_{n=1}^{m} \frac{b_{n}^{p}}{n-c}-\sum_{n=1}^{m}\left(\sum_{k=n}^{m} \frac{b_{k}}{(k-c)^{1+1 / p}}\right)^{p}
$$


and

$$
D_{i}=\left\{\left(b_{1}, b_{2}, \ldots, b_{n}\right) \mid 0 \leq \min _{1 \leq n \leq m}\left\{b_{n}\right\}<b_{i}=\max _{1 \leq n \leq m}\left\{b_{n}\right\}\right\}
$$

If $\left(b_{1}, b_{2}, \ldots, b_{n}\right) \in D_{i}$, then

$$
\begin{aligned}
\frac{\partial f}{\partial b_{i}} & =p^{p} \frac{p b_{i}^{p-1}}{i-c}-\frac{p}{(i-c)^{1+1 / p}} \sum_{n=1}^{i}\left(\sum_{k=n}^{m} \frac{b_{k}}{(k-c)^{1+1 / p}}\right)^{p-1} \\
& >\frac{p b_{i}^{p-1}}{(i-c)^{1+\frac{1}{p}}}\left[p^{p}(i-c)^{1 / p}-\sum_{n=1}^{i}\left(\sum_{k=n}^{m} \frac{1}{(k-c)^{1+1 / p}}\right)^{p-1}\right] \\
& >\frac{p b_{i}^{p-1}}{(i-c)^{1+\frac{1}{p}}}\left[p^{p}(i-c)^{1 / p}-\sum_{n=1}^{i}\left(\sum_{k=n}^{\infty} \frac{1}{(k-c)^{1+1 / p}}\right)^{p-1}\right] .
\end{aligned}
$$

By inequality (2.7), we know $\frac{\partial f}{\partial b_{i}}>0$. By Theorem B, inequality (3.2) holds, the proof is completed.

Corollary 1 If $p>1, m>0, m \in \mathrm{N}, a_{n}>0(n=1,2, \ldots, m), c \in\left(0, \frac{1}{2}\right)$ is the only one positive root of equation (2.5), and $B_{m}=\min _{1 \leq n \leq m}\left\{(n-c)^{1 / p} a_{n}\right\}$, then

$$
p^{p} \sum_{n=1}^{m} a_{n}^{p}-\sum_{n=1}^{m}\left(\sum_{k=n}^{m} \frac{a_{k}}{k-c}\right)^{p}>-p^{p} B_{m}^{p} \sum_{n=1}^{m} \frac{n^{p}-(n-c)^{p}}{(n-c)^{p+1}} .
$$

Proof By (3.1) and (2.8), we can obtain

$$
\begin{aligned}
p^{p} \sum_{n=1}^{m} a_{n}^{p}-\sum_{n=1}^{m}\left(\sum_{k=n}^{m} \frac{a_{k}}{k-c}\right)^{p} & >p^{p} B_{m}^{p}\left[\sum_{n=1}^{m} \frac{1}{n-c}-\sum_{n=1}^{m} \frac{n^{p}}{(n-c)^{p+1}}\right] \\
& =-p^{p} B_{m}^{p} \sum_{n=1}^{m} \frac{n^{p}-(n-c)^{p}}{(n-c)^{p+1}} .
\end{aligned}
$$

Corollary 2 If $p>1, a_{n}>0(n=1,2, \ldots), \sum_{n=1}^{\infty} a_{n}^{p}<+\infty$ and $c \in\left(0, \frac{1}{2}\right)$ is the only one positive root of equation (2.5), then

$$
\sum_{n=1}^{\infty}\left(\sum_{k=n}^{\infty} \frac{a_{k}}{k-c}\right)^{p} \leq p^{p} \sum_{n=1}^{\infty} a_{n}^{p}
$$

Proof Because of $\sum_{n=1}^{\infty} a_{n}^{p}<+\infty$, the infimum of $\left\{(n-c)^{1 / p} a_{n}\right\}_{n=1}^{\infty}$ is zero. Then there exists a sequence $\left\{m_{i} \mid m_{i} \in \mathrm{N}\right\}$ such that $\left\{\left(m_{i}-c\right)^{1 / p} a_{m_{i}}\right\}_{i=1}^{\infty}$ decrease to zero. Since (3.3), we have

$$
p^{p} \sum_{n=1}^{m_{i}} a_{n}^{p}-\sum_{n=1}^{m_{i}}\left(\sum_{k=n}^{m_{i}} \frac{a_{k}}{k-c}\right)^{p}>-p^{p}\left[\left(m_{i}-c\right)^{1 / p} a_{m_{i}}\right]^{p} \sum_{n=1}^{m_{i}} \frac{n^{p}-(n-c)^{p+1}}{(n-c)^{p+1}} .
$$

Let $i \rightarrow+\infty$ in inequality (3.5), we have $m_{i} \rightarrow+\infty$ and

$$
\lim _{i \rightarrow+\infty}\left[\left(m_{i}-c\right)^{1 / p} a_{m_{i}}\right]^{p} \sum_{n=1}^{m_{i}} \frac{n^{p}-(n-c)^{p+1}}{(n-c)^{p+1}}=0 .
$$


Then by (3.5), we can obtain

$$
p^{p} \sum_{n=1}^{m_{i}} a_{n}^{p}-\sum_{n=1}^{m_{i}}\left(\sum_{k=n}^{m_{i}} \frac{a_{k}}{k-c}\right)^{p} \geq 0
$$

Therefore, inequality (3.4) holds.

Remark Obviously, inequality (3.4) strengthens inequality (1.2).

\section{Strengthened Copson's inequality $(1 / 2<p<1)$}

Theorem 2 If $\frac{1}{2}<p<1, m>0, m \in \mathrm{N}, a_{n}>0(n=1,2, \ldots, m), d \in\left(0, \frac{1}{2}\right)$ is the only one positive root of equation (2.6) and $B_{m}=\min _{1 \leq n \leq m}\left\{(n+d)^{1 / p} a_{n}\right\}$. Then

$$
\sum_{n=1}^{m}\left(\sum_{k=n}^{m} \frac{a_{k}}{k+d}\right)^{p}-p^{p} \sum_{n=1}^{m} a_{n}^{p} \geq B_{m}^{p}\left[\sum_{n=1}^{m}\left(\sum_{k=n}^{m} \frac{1}{(k+d)^{1+1 / p}}\right)^{p}-p^{p} \sum_{n=1}^{m} \frac{1}{n+d}\right]
$$

Proof Let $b_{n}=(n+d)^{1 / p} a_{n}(n=1,2, \ldots, m)$. Then inequality (4.1) is equivalent to

$$
\sum_{n=1}^{m}\left(\sum_{k=n}^{m} \frac{b_{k}}{(k+d)^{1+1 / p}}\right)^{p}-p^{p} \sum_{n=1}^{m} \frac{b_{n}^{p}}{n+d} \geq B_{m}^{p}\left[p^{p} \sum_{n=1}^{m} \frac{1}{n}-\sum_{n=1}^{m}\left(\sum_{k=n}^{m} \frac{1}{k^{1+1 / p}}\right)^{p}\right]
$$

where $B_{m}=\min _{1 \leq n \leq m}\left\{b_{n}\right\}$. Set

$$
f: b \in(0,+\infty)^{m} \rightarrow \sum_{n=1}^{m}\left(\sum_{k=n}^{m} \frac{b_{k}}{(k+d)^{1+1 / p}}\right)^{p}-p^{p} \sum_{n=1}^{m} \frac{b_{n}^{p}}{n+d}
$$

and $D_{i}=\left\{\left(b_{1}, b_{2}, \ldots, b_{n}\right) \mid 0 \leq \min _{1 \leq n \leq m}\left\{b_{n}\right\}<b_{i}=\max _{1 \leq n \leq m}\left\{b_{n}\right\}\right\}$. If $\left(b_{1}, b_{2}, \ldots, b_{n}\right) \in D_{i}$, then

$$
\begin{aligned}
\frac{\partial f}{\partial b_{i}} & =\frac{p}{(i+d)^{1+1 / p}} \sum_{n=1}^{i}\left(\sum_{k=n}^{m} \frac{b_{k}}{(k+d)^{1+1 / p}}\right)^{p-1}-p^{p+1} \frac{b_{i}^{p-1}}{i+d} \\
& =\frac{p b_{i}^{p-1}}{(i+d)^{1+1 / p}}\left[\sum_{n=1}^{i}\left(\sum_{k=n}^{m} \frac{b_{k}}{(k+d)^{1+1 / p} b_{i}}\right)^{-(1-p)}-p^{p}(i+d)^{1 / p}\right] \\
& >\frac{p b_{i}^{p-1}}{(i+d)^{1+1 / p}}\left[\sum_{n=1}^{i}\left(\sum_{k=n}^{m} \frac{1}{(k+d)^{1+1 / p}}\right)^{-(1-p)}-p^{p}(i+d)^{1 / p}\right] \\
& >\frac{p b_{i}^{p-1}}{(i+d)^{1+1 / p}}\left[\sum_{n=1}^{i}\left(\sum_{k=n}^{\infty} \frac{1}{(k+d)^{1+1 / p}}\right)^{-(1-p)}-p^{p}(i+d)^{1 / p}\right]
\end{aligned}
$$

By Lemma 1, we have

$$
\begin{aligned}
\frac{\partial f}{\partial b_{i}}> & \frac{p b_{i}^{p-1}}{(i+d)^{1+1 / p}}\left[\sum_{n=1}^{i}\left(\int_{n-\frac{1}{2}}^{\infty} \frac{1}{(x+d)^{1+1 / p}} d x\right)^{-(1-p)}-p^{p}(i+d)^{1 / p}\right] \\
& =\frac{p b_{i}^{p-1}}{(i+d)^{1+1 / p}}\left[p^{-(1-p)} \sum_{n=1}^{i}\left(n-\frac{1}{2}+d\right)^{(1-p) / p}-p^{p}(i+d)^{1 / p}\right] .
\end{aligned}
$$


As $i=1$, by the definition of $d$, we have

$$
\frac{\partial f}{\partial b_{1}}>\frac{p b_{1}^{p-1}}{(1+d)^{1+1 / p}}\left[p^{-(1-p)}\left(\frac{1}{2}+d\right)^{(1-p) / p}-p^{p}(1+d)^{1 / p}\right]=0 .
$$

As $2 \leq i \leq m$, because $\frac{1}{2}<p<1,0<\frac{p-1}{p} \leq 1$ and $g: x \in(0,+\infty) \rightarrow x^{(1-p) / p}$ is concave, we have

$$
\begin{aligned}
\frac{\partial f}{\partial b_{i}}> & \frac{p b_{i}^{p-1}}{(i+d)^{1+1 / p}}\left[p^{-(1-p)}\left(\left(\frac{1}{2}+d\right)^{(1-p) / p}+\sum_{n=2}^{i}\left(n-\frac{1}{2}+d\right)^{(1-p) / p}\right)-p^{p}(i+d)^{1 / p}\right] \\
> & \frac{p b_{i}^{p-1}}{(i+d)^{1+1 / p}}\left[p ^ { - ( 1 - p ) } \left(\left(\frac{1}{2}+d\right)^{(1-p) / p}\right.\right. \\
& \left.\left.+\int_{\frac{3}{2}}^{i+\frac{1}{2}}\left(x-\frac{1}{2}+d\right)^{(1-p) / p} d x\right)-p^{p}(i+d)^{1 / p}\right] \\
= & \frac{p b_{i}^{p-1}}{(i+d)^{1+1 / p}}\left[p^{-(1-p)}\left(\left(\frac{1}{2}+d\right)^{(1-p) / p}+p(i+d)^{1 / p}-p(1+d)^{1 / p}\right)-p^{p}(i+d)^{1 / p}\right] \\
= & \frac{p b_{i}^{p-1}}{(i+d)^{1+1 / p}}\left[p^{-(1-p)} \cdot p(i+d)^{1 / p}-p^{p}(i+d)^{1 / p}\right]=0 .
\end{aligned}
$$

Thus, for every $D_{i}, \frac{\partial f}{\partial b_{i}}>0$. By Theorem B, inequality (4.2) holds.

Corollary 3 If $\frac{1}{2}<p<1, m>0, m \in \mathrm{N}, a_{n}>0(n=1,2, \ldots, m), d \in\left(0, \frac{1}{2}\right)$ is the only one positive root of equation (2.6) and $B_{m}=\min _{1 \leq n \leq m}\left\{(n+d)^{1 / p} a_{n}\right\}$. Then

$$
\sum_{n=1}^{m}\left(\sum_{k=n}^{m} \frac{a_{k}}{k+d}\right)^{p}-p^{p} \sum_{n=1}^{m} a_{n}^{p} \geq p^{p} B_{m}^{p} \sum_{n=1}^{m} \frac{n^{p}-(n+d)^{p}}{(n+d)^{p+1}}
$$

Proof From Theorem 2 and Lemma 5, we have

$$
\sum_{n=1}^{m}\left(\sum_{k=n}^{m} \frac{a_{k}}{k+d}\right)^{p}-p^{p} \sum_{n=1}^{m} a_{n}^{p} \geq B_{m}^{p}\left[p^{p} \sum_{n=1}^{m} \frac{n^{p}}{(n+d)^{p+1}}-p^{p} \sum_{n=1}^{m} \frac{1}{n+d}\right]
$$

Then inequality (4.3) holds.

Corollary 4 If $\frac{1}{2}<p<1, a_{n}>0(n=1,2, \ldots), d \in\left(0, \frac{1}{2}\right)$ is the only one positive root of equation (2.6) and series $\sum_{n=1}^{\infty}\left(\sum_{k=n}^{\infty} \frac{a_{k}}{k+d}\right)^{p}<+\infty$. Then

$$
\sum_{n=1}^{\infty}\left(\sum_{k=n}^{\infty} \frac{a_{k}}{k+d}\right)^{p} \geq p^{p} \sum_{n=1}^{\infty} a_{n}^{p}
$$

Proof According to inequality (4.3), we obtain

$$
\sum_{n=1}^{m}\left(\sum_{k=n}^{m} \frac{a_{k}}{k+d}\right)^{p}+p^{p} B_{m}^{p} \sum_{n=1}^{m} \frac{(n+d)^{p}-n^{p}}{(n+d)^{p+1}} \geq p^{p} \sum_{n=1}^{m} a_{n}^{p}
$$

The following proof is the same as the relevant proof for Corollary 2, omitted here. 
Remark For $\frac{1}{2}<p<1$, there is no doubt that inequality (4.4) strengthens inequality (1.3).

\section{Competing interests}

The author declares that they have no competing interests.

\section{Acknowledgements}

The research is supported by the Nature Science Foundation of China (No. 110771069) and the NS Foundation of the Educational Committee of Zhejiang Province under Grant Y201223283.

Received: 11 July 2012 Accepted: 28 November 2012 Published: 19 December 2012

\section{References}

1. Hardy, GH, Littlewood, JE, Poly, AG: Inequalities. Cambridge University Press, Cambridge (1952)

2. Yang, B-C: Arithmetic Operators and Hilbert Type Inequality. Science Press, Beijing (2009) (in Chinese)

3. Kuang, J-C: Inequality of Regular. Shandong Science and Technology Press, Jinan (2004) (in Chinese)

4. Pachpatte, BG: Mathematical Inequalities, pp. 118-127. Elsevier, Amsterdam (2005)

5. Bullen, PS: A Dictionary of Inequalities, p. 65. Chapman \& Hall, London (1998)

6. Gao, P: On weighted remainder form of Hadry-type inequalities. RGMIA 12(3), 17-32 (2009) (http://www.staff.vu.edu.au/RGMIA/v12n3.asp)

7. Zhang, Xiao-Ming, Chu, Yu-Ming: A new method to study analytic inequalities. J. Inequal. Appl. 2010, Article ID $698012(2010)$

doi:10.1186/1029-242X-2012-305

Cite this article as: Xu: On strengthened form of Copson's inequality. Journal of Inequalities and Applications 2012 2012:305.

\section{Submit your manuscript to a SpringerOpen ${ }^{\ominus}$ journal and benefit from:}

- Convenient online submission

Rigorous peer review

- Immediate publication on acceptance

- Open access: articles freely available online

- High visibility within the field

- Retaining the copyright to your article 